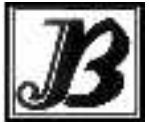

J. bio-sci. 21: 99-108, 2013

ISSN 1023-8654

http://www.banglajol.info/index.php/JBS/index

\title{
ISOLATION AND IDENTIFICATION OF BACTERIA AND THEIR DRUG SUSCEPTIBILITY PATTERN IN CHILDREN TO ESTABLISH THE CORRELATION BETWEEN CHILDHOOD SEPTICEMIA WITH C-REACTIVE PROTEIN (CRP) LEVELS
}

\author{
Al Jahidi Hasan Chowdhurya ${ }^{*}$, M Shahjahana, Tanzima Yeasmin ${ }^{b}$, Mohammad Shariar Shovona, \\ M Mushfequr Rahmanc
}

aDepartment of Biochemistry and Molecular Biology, University of Rajshahi,

bInstitute of Biological Sciences, University of Rajshahi,, c University of Health Sciences, Dhaka, Bangladesh

\begin{abstract}
Context: Neonatal sepsis is one of the most important causes of morbidity and mortality and C-reactive protein (CRP) an excellent biomarker has significant diagnostic and prognostic value for the treatment of septicemia patient.

Objectives: To isolate and identify viable pathogens from blood culture and their antibiogram and to correlate CRP levels with septicemic child.

Materials and Methods: A total of 273 cases among which 233 were clinically suspected septicemia cases and 40 were healthy controls in age group 0 day to 15 years were selected from United Hospital Ltd., Dhaka Bangladesh. Blood culture was analyzed by the instrument BACTEC 9120 series. CRP was measured from blood serum by the auto biochemical analyzer OLYMPUS AU 640 followed by immuno-turbidimetric method. The organisms were isolated by inoculation on blood agar and MacConkey agar media. Identification of the organisms was done by colony morphology, gram staining and biochemical tests. Sensitivity of isolates was done against antimicrobial agents by disc diffusing method.

Results: Blood samples in total 233 cases of suspected septicemia in children were studied between the ages of 0 day to 15 years. Culture proven septicemia 39 (16.74\%), probable septicemia $136(58.37 \%)$ and nonsepticemic febrile patients 58 (24.90\%) were found. The highest rate of blood culture positivity found among 5-10 yrs. age group (25.64\%). Salmonella typhi (41.03\%) was the most common infective agent. The rate of blood culture positivity was significantly higher $(p<0.001)$ among patients without antimicrobial therapy $(23.74 \%)$ than those in patients with antimicrobial therapy (6.38\%). S. typhi were $75 \%$ sensitive to Ceftriaxone while azithromycin showed high rate resistance $(85.71 \%)$. E. coli and Klebsiella pneumoniae were highly sensitive $(100 \%)$ to imipenem but $E$. coli resistant $(100 \%)$ to amikacin, amoxyclavonic acid ciprofloxacin, gentamicin, Cefepime and netilmicin. Resistant (100\%) to gentamicin was observed from K. pneumoniae. Mean CRP values $(\mathrm{mg} / \mathrm{l})$ of blood culture proven septicemia group, probable septicemia group, non-septicemic febrile group and control group were $70.42,34.05,3.08$ and 0.98 respectively. Both proven septicemia and suspected septicemia cases showed CRP concentration above the cut-off value $(>6 \mathrm{mg} / \mathrm{l})$ and $p$ value significant $(p<0.001)$. Statistically significant difference $(p<0.001)$ was found when mean CRP level of proven septicemia group, probable septicemia group and non-septicemic febrile group each compared with control group. CRP concentration were significantly $(p<0.001)$ different among three study group.

Conclusion: Most of the gram negative bacteria isolated from blood culture showed resistance to commonly used antibiotics. The predominant infective isolate was Salmonella typhi. In this study, CRP level is high (cut-off value $6 \mathrm{mg} / \mathrm{l}$ ) both in proven and probable septicemia group. CRP may have a good biomarker tools in diagnostic and prognostic value. Investigation of blood culture should be done before antimicrobial therapy,
\end{abstract}

Key words: Septicemia, C Reactive Protein (CRP), Multi drug resistant (MDR), Sensitive, Resistant, Blood culture. Introduction

Septicemia is a clinical state in which bacteria are present in blood stream and gives rise to serious systemic symptoms (Murphy 1988). Sepsis among children is a significant health problem and is a leading cause of death of children worldwide (Watson et al. 2005). Detection of bacteria in blood is critically important because

*Corresponding author Email: ajhchy@yahoo.com 
septicemia has mortality as high as 50\% (Washington and Ilstrup 1986). Early initiation of treatment with appropriate antimicrobial agents is essential to reduce mortality (Weinstein et al. 1983, Kreger et al. 1980). Blood culture remains the ideal method for the diagnosis and treatment of patients with suspected septicemia (Spencer 1988, Pierce and Murray 1986).Microbial invasion of blood stream causes of high rates of morbidity and mortality (Laupland et al. 2004). Study report from Dhaka Shishu hospital revealed that blood culture proven septicemia was 35\% (Ahmed et al. 2002). A study in Khulna Medical College hospital on neonatal infection shown septicemia (34.6\%) was the commonest major infection (Rasul et al. 2007).

Only few data is available regarding etiology of septicemia of children and their antimicrobial sensitivity pattern in Bangladesh. So it is of great importance to know the susceptibility profile of the whole range of likely pathogens in order to select appropriate antibiotics (Esel et al. 2003). Therefore, this study has been designed to identify the etiological agents of septicemia in children and to determine antimicrobial susceptibility pattern and to establish correlation for usefulness of CRP as biomarker for the septicemic children.

\section{Materials and Methods}

A total 273 cases among which 233 were clinically suspected septicemia cases and 40 were healthy controls in age group 0 day to 15 years were selected from United Hospital Ltd. (UHL), Gulshan, Dhaka, Bangladesh. Blood culture was analyzed by the instrument BACTEC 9120 series. CRP was measured from blood serum by the auto biochemical analyzer OLYMPUS AU 640 followed by immuno-turbidimetric method.

Microbiological Methods: The organisms were isolated from specimen by inoculation subculture on blood agar and MacConkey agar media. Identification of the organisms was done by colony morphology, gram staining and standard biochemical tests. All the isolates were tested for sensitivity against antimicrobial agents by disc diffusing method of Kirby Bauer et al. (1966). The potency of each batch of disc was standardized by the reference strain of ATCC Esch. Coli, No 25922 and Pseudomonas aeruginosa No 27853. Zone of inhibition were compared with the standard value and was considered as sensitive (S), Intermediate sensitive (IS) and resistant (R) according to the NCCLS (1998).

Data processing and analysis:

The daily collected data were checked, verified and edited daily. Data were coded and entered into computer by using SPSS data entry II program. Statistical significance was tested with appropriate tests.

The study patients were grouped as follows (age range 0-15 years):

\section{A. According to clinical features, Blood culture result and CRP concentration in blood (Bont et al.} 1994).

1. Patients with clinical suspicion of sepsis, positive blood culture and CRP concentration $>6 \mathrm{mg} / \mathrm{l}$ were included in culture proven group.

2. Patients with clinical suspicion of sepsis but without bacteriological confirmation and $\mathrm{CRP}>6 \mathrm{mg} / \mathrm{l}$ were included in probable septicemia group.

3. Patients with clinical suspicion of sepsis but without bacteriological confirmation and $\mathrm{CRP}<6 \mathrm{mg} / \mathrm{l}$ were included in non-septicemia febrile group.

4. The control group comprised age and sex matched children without history of fever or any other inflammatory and immunological illness for last 3 months.

\section{B. According to antibiotic therapy}

1. Patients with anti-microbial therapy: Patients who were receiving antimicrobial agents within 48 hours of taking blood samples for culture.

2. Patients without anti-microbial therapy 


\section{Results}

Among 233 study cases 123 were male and 110 were female. The highest number $71(30.47 \%)$ of patients were 0 day-1 month age group followed by $48(20.60 \%)$ from 1 mon.-1 year, 44(18.88\%) from 1-5 years of age group. 5-10 yrs. and 10-15 yrs. age group, there were 40(17.17\%) and 30(12.88\%) patients respectively in each group. The highest rate of blood culture positively 10(25\%) were found among patients of 5-10 yrs. age group where lowest rate positivity 5(10.42\%) were found between 1 mon.-1 yrs. age group. Blood culture was positive in 39(16.74\%) study cases. Out of 123 male patients, blood culture were positive in 22(17.89\%) and out of 110 female patient's blood culture were positive in 17 (15.45\%) patients. No significant difference were observed between sex groups ( $P>0.05)$. Figure 1 shows Salmonella typhi 16(41.03\%) was the most common predominance isolates followed by $S$. paratyphi A 7(17.95\%). Klebsiella pneumoniae and Acinetobacter baumannii were same 4(10.26\%) whereas Pseudomonas spp. 3(7.69\%), Serratia marcescens 2(5.13\%). S. aureus 1(2.56\%), E. coli 1(2.56\%) and Enterococcus spp. 1(2.56\%) were isolated.

Both blood culture and CRP were positive in 39 (16.74\%) cases, only CRP were positive in 136 (58.37\%) and both blood culture and CRP level were negative in $58(24.89 \%)$ cases. The rate of blood culture positivity was significantly higher $(p<0.001)$ among patients without antimicrobial therapy $(23.74 \%)$ than those in patients with antimicrobial therapy (6.38\%).

Table 1. Mean CRP level in blood and mean age among different groups of study cases.

\begin{tabular}{|c|c|c|c|c|c|}
\hline \multirow[t]{2}{*}{ Parameters } & \multicolumn{4}{|c|}{ Study different group } & \multirow[t]{2}{*}{$P$-value } \\
\hline & $\begin{array}{ll}\text { Culture } & \text { proven } \\
\text { Septicemia } & \end{array}$ & Probable Septicemia & $\begin{array}{l}\text { Non-septicemic febrile } \\
\text { group }\end{array}$ & Control group & \\
\hline No. of subjects & 39 & 136 & 58 & 40 & \\
\hline \multicolumn{6}{|l|}{ Male/female } \\
\hline Age (days) & $2098.23 \pm 1860.09$ & $1093.75 \pm 1484.79$ & $1011.44 \pm 1427.67$ & $1631.8 \pm 1681.04$ & $\begin{array}{l}0.810^{a} \\
0.358^{b} \\
0.306^{c}\end{array}$ \\
\hline $\mathrm{CRP}(\mathrm{mg} / \mathrm{l})$ & $70.42 \pm 43.25$ & $34.05 \pm 18.95$ & $3.08 \pm 1.29$ & $0.98 \pm 0.45$ & $\begin{array}{l}<0.001^{a}, \\
<0.001^{b} \\
<0.001^{c}\end{array}$ \\
\hline $\mathrm{CRP}(\mathrm{mg} / \mathrm{l})$ & $70.42 \pm 43.25$ & $34.05 \pm 18.95$ & $3.08 \pm 1.29$ & & $\begin{array}{l}<0.001^{d}, \\
<0.001^{e} \\
<0.001^{f}\end{array}$ \\
\hline
\end{tabular}

$p$-value consider significant at $p<0.05$. $p$-values are from one way ANOVA test.

Data are presented as mean $\pm \mathrm{SD}$.

a $p$-value comparison between control and Culture proven septicemia;

${ }^{b} p$-value comparison between control and Probable Septicemia;

c $p$-value comparison between control and Non-septicemic febrile group.

d $p$-value comparison between Culture proven septicemia and Probable Septicemia ;

e $p$-value comparison between Culture proven septicemia and Non-septicemic febrile group;

f $p$-value comparison between Probable Septicemia and Non-septicemic febrile group.

Table 1 shows that mean CRP values (mg/l) of blood culture proven septicemia group, probable septicemia group, non-septicemic febrile group and control group were $70.42,34.05,3.08$ and 0.98 respectively. Statistically significant differences $(p<0.001)$ were found when mean CRP level of proven septicemia group, probable septicemia group and non-septicemic febrile group each compared with control group. 
The average age (mean $\pm S D$ ) of the culture proven septicemia, probable septicemia, non-septicemic febrile and control groups were $2098.23 \pm 1860.09,1093.75 \pm 1484.79,1011.44 \pm 1427.67$ and $1631.8 \pm 1681.04$ days respectively. No significant differences $(p>0.05)$ among study groups were found in respect to age. CRP concentration were found highly positive association $(p<0.001$ ) when compared among three different study groups.

Table 2. Blood culture positivity among patients with or without antimicrobial therapy.

\begin{tabular}{|l|l|l|l|}
\hline Categories of patients & No. of patients & No. of positive blood culture. & No. of negative blood culture \\
\hline Without antimicrobial & 139 & $33(23.74 \%)$ & $106(76.26 \%)$ \\
\hline With antimicrobial & 94 & $6(6.38 \%)$ & $88(93.62 \%)$ \\
\hline Total & 233 & $39(16.74 \%)$ & $194(83.26 \%)$ \\
\hline
\end{tabular}

$(P<0.001$. Significantly higher positivity rate among patients without antimicrobial therapy. $p$ - values are from one way ANOVA test.)

Table 2 shows out of 233 blood samples cultured, 139 were from patients without antimicrobial therapy and 94 were with antimicrobial therapy. Patients, without antimicrobial therapy, blood culture were positive in 33 $(23.74 \%)$ and with antimicrobial therapy was positive in $6(6.38 \%)$ patients. The difference was statistically significant $(p<0.001)$.

Fig.1. Distribution of bacteria isolated from Blood .

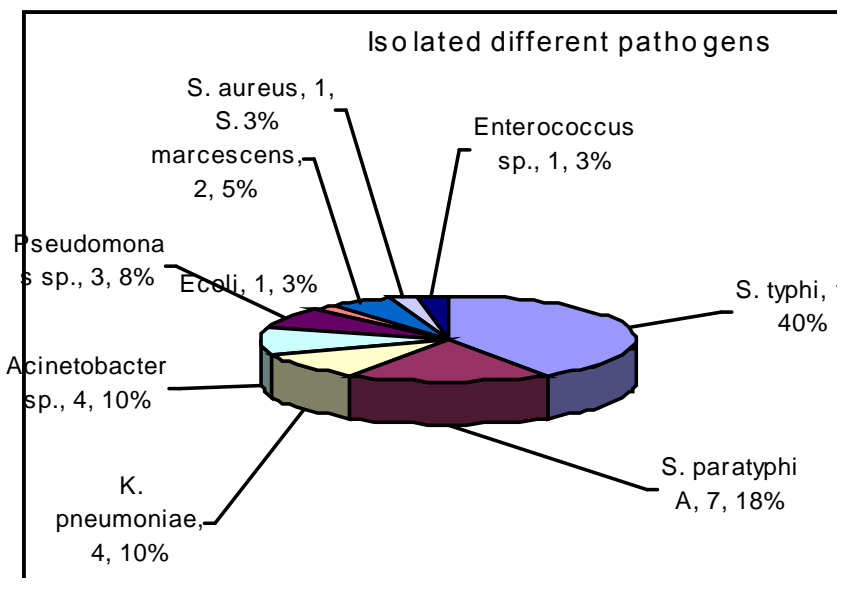


Table 3. Sensitivity pattern of isolated Pathogens against different antimicrobial agents.

\begin{tabular}{|c|c|c|c|c|c|c|c|c|c|c|}
\hline \multirow{2}{*}{$\begin{array}{l}\text { Antimic } \\
\text { robial } \\
\text { agent }\end{array}$} & \multirow[t]{2}{*}{$\begin{array}{l}\text { Sensitivity } \\
\text { pattern }\end{array}$} & \multicolumn{9}{|c|}{ Name of bacterial isolates } \\
\hline & & $\begin{array}{l}\text { S. typhi } \\
(n=16)\end{array}$ & $\begin{array}{l}\text { S. } \\
\text { paratyphi A } \\
(\mathrm{n}=07)\end{array}$ & $\begin{array}{l}K . \\
\text { pneumoniae } \\
(\mathrm{n}=04)\end{array}$ & $\begin{array}{l}\text { E. coli } \\
(\mathrm{n}=01)\end{array}$ & $\begin{array}{l}S \text {. } \\
\text { marcescen } \\
s(n=02)\end{array}$ & $\begin{array}{l}\text { S. aureus } \\
(\mathrm{n}=01)\end{array}$ & $\begin{array}{c}\text { Enterococcu } \\
\text { s sp. }(n=3)\end{array}$ & $\begin{array}{l}\text { Pseudomonas } \\
\text { sp. }(n=3)\end{array}$ & $\begin{array}{l}\text { Acinetobacter } \\
\text { baumannii( }(n= \\
\text { 4) }\end{array}$ \\
\hline AMC & $\begin{array}{l}\mathrm{S} \\
\mathrm{IS} \\
\mathrm{R}\end{array}$ & - & - & $\begin{array}{l}1(25.00 \%) \\
1(25.00 \%) \\
2(50.00 \%)\end{array}$ & $\begin{array}{l}0(00) \\
0(00) \\
1(100 \%)\end{array}$ & $\begin{array}{l}0(00) \\
1(50.00 \%) \\
1(50.00 \%)\end{array}$ & $\begin{array}{l}0(00) \\
0(00) \\
1(100 \%)\end{array}$ & $\begin{array}{l}0(00) \\
0(00) \\
1(100 \%)\end{array}$ & - & $\begin{array}{l}0(00) \\
0(00) \\
4(100 \%)\end{array}$ \\
\hline AMP & $\begin{array}{l}\mathrm{S} \\
\mathrm{IS} \\
\mathrm{R}\end{array}$ & $\begin{array}{l}9(56.25 \%) \\
0(00) \\
7(43.75 \%)\end{array}$ & $\begin{array}{l}3(42.86 \%) \\
0(00) \\
4(57.14 \%)\end{array}$ & - & - & - & - & - & . & - \\
\hline AZM & $\begin{array}{l}S \\
\text { IS } \\
R\end{array}$ & $\begin{array}{l}3(18.75 \%) \\
1(6.25 \%) \\
12(75.00 \%)\end{array}$ & $\begin{array}{l}1(14.29 \%) \\
0(00) \\
6(85.71))\end{array}$ & - & - & - & . & - & - & - \\
\hline CRO & $\begin{array}{l}S \\
\text { IS } \\
R\end{array}$ & $\begin{array}{l}12(75 \%) \\
0(00) \\
4(25 \%)\end{array}$ & $\begin{array}{l}5(71.43 \%) \\
0(00) \\
2(28.57 \%)\end{array}$ & $\begin{array}{l}1(25.00 \%) \\
0(00) \\
3(75.00 \%)\end{array}$ & $\begin{array}{l}1(100 \%) \\
0(00) \\
0(00)\end{array}$ & $\begin{array}{l}0(00) \\
1(50.00 \%) \\
1(50.00 \%)\end{array}$ & - & - & $\begin{array}{l}0(00) \\
0(00) \\
3(100 \%)\end{array}$ & $\begin{array}{l}2(50.00 \%) \\
1(25.00 \%) \\
1(25.00 \%)\end{array}$ \\
\hline c & $\begin{array}{l}S \\
\text { IS } \\
R\end{array}$ & $\begin{array}{l}8(50.00 \%) \\
0(00) \\
8(50.00 \%)\end{array}$ & $\begin{array}{l}3(42.85 \%) \\
1(14.29 \%) \\
3(42.86 \%)\end{array}$ & - & - & - & - & - & - & - \\
\hline SXT & $\begin{array}{l}S \\
\text { IS } \\
R\end{array}$ & $\begin{array}{l}7(43.75 \%) \\
0(00) \\
9(56.25 \%)\end{array}$ & $\begin{array}{l}3(42.86 \%) \\
0(00) \\
4(57.14 \%)\end{array}$ & $\begin{array}{l}0(00) \\
1(25.00 \%) \\
3(75.00 \%)\end{array}$ & $\begin{array}{l}0(00) \\
1(100 \%) \\
0(00)\end{array}$ & $\begin{array}{l}2(100 \%) \\
0(00) \\
0(00)\end{array}$ & $\begin{array}{l}0(00) \\
1(100 \%) \\
0(00)\end{array}$ & $\begin{array}{l}0(00) \\
1(100 \%) \\
0(00)\end{array}$ & $\begin{array}{l}0(00) \\
0(00) \\
3(100 \%)\end{array}$ & $\begin{array}{l}2(50.00 \%) \\
1(25.00 \%) \\
1(25.00 \%)\end{array}$ \\
\hline CIP & $\begin{array}{l}S \\
\text { IS } \\
R\end{array}$ & $\begin{array}{l}10(62.50 \%) \\
2(12.50 \%) \\
4(25.00 \%)\end{array}$ & $\begin{array}{l}4(57.14 \%) \\
0(00) \\
3(42.86 \%)\end{array}$ & $\begin{array}{l}1(25.00 \%) \\
0(00) \\
3(75.00 \%)\end{array}$ & $\begin{array}{l}0(00) \\
0(00) \\
1(100 \%)\end{array}$ & $\begin{array}{l}0(00) \\
1(50.00 \%) \\
1(50.00 \%)\end{array}$ & $\begin{array}{l}0(00) \\
1(100 \%) \\
0(00)\end{array}$ & $\begin{array}{l}0(00) \\
0(00) \\
1(100 \%)\end{array}$ & $\begin{array}{l}2(66.67 \%) \\
1(33.33 \%) \\
0(00)\end{array}$ & $\begin{array}{l}3(75.00 \%) \\
0(00) \\
1(25.00 \%)\end{array}$ \\
\hline FEP & $\begin{array}{l}S \\
\text { IS } \\
R\end{array}$ & $\begin{array}{l}10(62.50 \%) \\
0(00) \\
6(37.50 \%)\end{array}$ & $\begin{array}{l}5(71.43 \%) \\
0(00) \\
2(28.57 \%)\end{array}$ & $\begin{array}{l}2(50.00 \%) \\
0(00) \\
2(50.00 \%)\end{array}$ & $\begin{array}{l}0(00) \\
0(00) \\
1(100 \%)\end{array}$ & $\begin{array}{l}2(100 \%) \\
0(00) \\
0(00)\end{array}$ & $\begin{array}{l}0(00) \\
0(00) \\
1(100 \%)\end{array}$ & $\begin{array}{l}1(100 \%) \\
0(00) \\
0(00)\end{array}$ & $\begin{array}{l}2(66.67 \%) \\
0(00) \\
1(33.33 \%)\end{array}$ & $\begin{array}{l}1(25.00 \%) \\
0(00) \\
3(75.00 \%)\end{array}$ \\
\hline CFM & $\begin{array}{l}\mathrm{S} \\
\mathrm{IS} \\
\mathrm{R}\end{array}$ & $\begin{array}{l}8(50.00 \%) \\
16.25 \% \\
7(43.75 \%)\end{array}$ & $\begin{array}{l}4(57.14 \%) \\
0(00) \\
3(42.86 \%)\end{array}$ & $\begin{array}{l}1(25.00 \%) \\
0(00) \\
3(75.00 \%)\end{array}$ & $\begin{array}{l}0(00) \\
1(100 \%) \\
0(00)\end{array}$ & $\begin{array}{l}0(00) \\
0(00) \\
2(100 \%)\end{array}$ & $\begin{array}{l}0(00) \\
0(00) \\
1(100 \%)\end{array}$ & $\begin{array}{l}0(00) \\
0(00) \\
1(100 \%)\end{array}$ & $\begin{array}{l}0(00) \\
0(00) \\
3(100 \%)\end{array}$ & . \\
\hline AK & $\begin{array}{l}S \\
\text { IS } \\
R\end{array}$ & - & - & $\begin{array}{l}3(75.00 \%) \\
0(00) \\
1(25.00 \%)\end{array}$ & $\begin{array}{l}0(00) \\
0(00) \\
1(100 \%)\end{array}$ & $\begin{array}{l}1(50.00 \%) \\
1(50.00 \%) \\
0(00)\end{array}$ & - & - & $\begin{array}{l}3(100 \%) \\
0(00) \\
0(00)\end{array}$ & $\begin{array}{l}2(50.00 \%) \\
0(00) \\
2(50.00 \%)\end{array}$ \\
\hline $\mathrm{CN}$ & $\begin{array}{l}\text { S } \\
\text { IS } \\
\mathrm{R}\end{array}$ & - & - & $\begin{array}{l}0(00) \\
0(00) \\
4(100 \%)\end{array}$ & $\begin{array}{l}0(00) \\
0(00) \\
1(100 \%)\end{array}$ & $\begin{array}{l}0(00) \\
0(00) \\
2(100 \%)\end{array}$ & - & - & $\begin{array}{l}1(33.33 \%) \\
1(33.33 \%) \\
1(33.33 \%)\end{array}$ & $\begin{array}{l}1(25.00 \%) \\
0(00) \\
3(75.00 \%)\end{array}$ \\
\hline
\end{tabular}


Table 3. Sensitivity pattern of isolated Pathogens against different antimicrobial agents. (Contd.)

\begin{tabular}{|c|c|c|c|c|c|c|c|c|c|c|}
\hline \multirow{2}{*}{$\begin{array}{l}\text { Antimic } \\
\text { robial } \\
\text { agent }\end{array}$} & \multirow[t]{2}{*}{$\begin{array}{l}\text { Sensitivity } \\
\text { pattern }\end{array}$} & \multicolumn{9}{|c|}{ Name of bacterial isolates } \\
\hline & & $\begin{array}{l}\text { S. typhi } \\
(n=16)\end{array}$ & $\begin{array}{l}\text { S. } \\
\text { paratyphi A } \\
(\mathrm{n}=07)\end{array}$ & $\begin{array}{l}\text { K. } \\
\text { pneumoniae } \\
(n=04)\end{array}$ & $\begin{array}{l}\text { E. coli } \\
(\mathrm{n}=01)\end{array}$ & $\begin{array}{l}\text { S. } \\
\text { marcescen } \\
s(n=02)\end{array}$ & $\begin{array}{l}\text { S. aureus } \\
(\mathrm{n}=01)\end{array}$ & $\begin{array}{l}\text { Enterococcu } \\
\text { s sp. }(n=3)\end{array}$ & $\begin{array}{l}\text { Pseudomonas } \\
\text { sp. }(n=3)\end{array}$ & $\begin{array}{l}\text { Acinetobacter } \\
\text { baumanni( }(n= \\
\text { 4) }\end{array}$ \\
\hline IPM & $\begin{array}{l}S \\
\text { IS } \\
R\end{array}$ & - & - & $\begin{array}{l}4(100 \%) \\
0(00) \\
0(00)\end{array}$ & $\begin{array}{l}1(100 \%) \\
0(00) \\
0(00)\end{array}$ & $\begin{array}{l}2(100 \%) \\
0(00) \\
0(00)\end{array}$ & - & - & $\begin{array}{l}0(00) \\
0(00) \\
3(100 \%)\end{array}$ & $\begin{array}{l}3(75.00 \%) \\
0(00) \\
1(25.00 \%)\end{array}$ \\
\hline NET & $\begin{array}{l}\text { S } \\
\text { IS } \\
\text { R }\end{array}$ & - & - & $\begin{array}{l}1(25.00 \%) \\
1(25.00 \%) \\
2(50.00 \%)\end{array}$ & $\begin{array}{l}0(00) \\
0(00) \\
1(100 \%)\end{array}$ & $\begin{array}{l}1(50.00 \%) \\
0(00) \\
1(50.00 \%)\end{array}$ & - & - & - & - \\
\hline DO & $\begin{array}{l}S \\
\text { IS } \\
R\end{array}$ & - & - & - & - & - & $\begin{array}{l}0(00) \\
0(00) \\
1(100 \%)\end{array}$ & $\begin{array}{l}0(00) \\
0(00) \\
1(100 \%)\end{array}$ & - & - \\
\hline LZD & $\begin{array}{l}S \\
\text { IS } \\
R\end{array}$ & - & - & - & - & - & $\begin{array}{l}1(100 \%) \\
0(00) \\
0(00)\end{array}$ & $\begin{array}{l}1(100 \%) \\
0(00) \\
0(00)\end{array}$ & - & - \\
\hline $0 x$ & $\begin{array}{l}\text { S } \\
\text { IS } \\
R\end{array}$ & - & - & - & - & - & $\begin{array}{l}1(100 \%) \\
0(00) \\
0(00)\end{array}$ & . & - & - \\
\hline $\mathrm{P}$ & $\begin{array}{l}S \\
\text { IS } \\
R\end{array}$ & - & - & - & - & - & $\begin{array}{l}0(00) \\
0(00) \\
1(100 \%)\end{array}$ & $\begin{array}{l}0(00) \\
0(00) \\
1(100 \%)\end{array}$ & - & - \\
\hline VA & $\begin{array}{l}\text { S } \\
\text { IS } \\
\text { R }\end{array}$ & . & . & - & - & . & $\begin{array}{l}1(100 \%) \\
0(00) \\
0(00)\end{array}$ & $\begin{array}{l}1(100 \%) \\
0(00) \\
0(00)\end{array}$ & . & . \\
\hline CAZ & $\begin{array}{l}\text { S } \\
\text { IS } \\
\text { R }\end{array}$ & - & - & - & - & - & - & - & $\begin{array}{l}0(00) \\
1(33.33 \%) \\
2(66.67 \%)\end{array}$ & $\begin{array}{l}0(00) \\
1(25.00 \%) \\
3(75.00 \%)\end{array}$ \\
\hline TZP & $\begin{array}{l}S \\
\text { IS } \\
R\end{array}$ & - & - & - & - & - & - & - & $\begin{array}{l}3(100 \%) \\
0(00) \\
0(00)\end{array}$ & - \\
\hline
\end{tabular}

AMC- Amoxyclavonic acid, AMP-Ampicillin, AZM-Azithromycin, CRO-Ceftriaxone, C-Chloramphenicol, SXT-Cotrimoxazole, CIP- Ciprofloxacin, FEPCefepime, CFM- Cefixime, AK- Amikacin, CN- Gentamicin, IPM- Imipenem, NET- Netilmicin, DO- Doxycycline, LZD- Linezolid, OX- Oxacillin, P- Penicillin, VA-Vancomycin. TZP-Piperacillin-Tazobactam, CAZ-Ceftazidime, S-Sensitive, IS- Intermediate sensitive, R- Resistant.

Table 3 showed S. typhi were $75 \%$ sensitive to ceftriaxone followed by ciprofloxacin and cefepime $62.50 \%$ each. $75 \%$ highly resistance was noted to azithromycin. K. pneumoniae were found $100 \%$ sensitive to imipenem, $75 \%$ to amikacin and cefepime. On the other hand, 100\% resistance against gentamicin. E. coli were sensitive (100\%) to Ceftriaxone and imipenem but were 100\% resistant to amikacin, amoxyclavonic acid ciprofloxacin, gentamicin and Cefepime. S. marcescens were 100\% sensitive to cotrimoxazole, cefepime and imipenem whereas $100 \%$ resistant to cefixime and gentamicin. On the other hand, Pseudomonas spp. were 100\% sensitive to amikacin and piperacillin-tazobactam but 100\% each resistant to ceftriaxone, cotrimoxazole, imipenem and cefixime. A. baumannii were $75 \%$ sensitive to ciprofloxacin \& imipenem each and resistance 100\% against amoxyclavonic acid. S. aureus was 100\% sensitive to lynezolid, oxacillin and vancomycin but $100 \%$ resistance were revealed to ampicillin, cefepime, cefixime, doxycycline and penicillin. Enterococcus sp. 100\% resistant to ampicillin, ciprofloxacin, cefixime doxycycline, and penicillin. 


\section{Discussion}

Out of 233 with clinically suspected septicemia cases, 39 (16.74\%) yielded bacterial growth. Similarly in BSMMU, Dewanjee (2000) have reported $26.08 \%$ of septicemia among pediatrics age groups. In Bangladesh, Saha et al., (2001) have isolated aerobic bacteria in $23.5 \%$ cases of septicemia in children. Among blood culture positive cases 22 (17.89\%) were male and 17(15.45\%) female $(P>0.05)$.

Among 39 isolates, S. typhi was $41.03 \%$, followed by S. paratyphi A (17.95\%). K. pneumoniae and A. baumannii were $(10.26 \%)$ in each. Next to Pseudomonas spp. (7.69\%), S. marcescens (5.13\%), S. aureus (2.56\%), E. coli (2.56\%), and finally Enterococcus sp. (2.56\%) were revealed in our study. Our results are in agreement with those of Sharma et al. (2006) in Nepal, Cheng et al. (1991) in Hongkong and Blomberg et al. (2007) in Tanzania. In an earlier study in Bangladesh, Dhaka Medical College, Begum et al. (2007) have found 22.03\% Salmonella spp. in neonates. In Bangladesh, Brooks et al. (2005) also have found S. typhi the common pathogen (75.4\%) isolated from blood culture. Another study in Niegeria, (Abuceju and Capeding, 2001) reported $15.9 \%$ isolation rates of Salmonella spp. from blood culture. Septicemia due to Salmonella spp. was predominant in children of Bangladesh because of living in unhygienic conditions, lack of sanitation facilities and taking of unhygienic foods and polluted water. In contrast to our findings, in Bangladesh, Ahmed et al. (2002), from Dhaka Shishu Hospital revealed that the principal organisms were E. coli (30\%) followed by Klebsiella spp., S. aureus, pseudomonas spp., Streptococcus spp. and Acinetobacter spp. Another study in 1996 from U.S.A. reported the most common blood stream isolates were S. aureus, K. pneumoniae, E. coli, Coagulase negative Staphylococcus and Pseudomonas spp. These different findings in USA may be due to striking geographical difference, increased use of invasive procedures, extensive surgery, intravascular devices and increase in the number of immunocompromized persons.

In our study, $6.38 \%$ of patients showed positive blood culture among the patients already on antimicrobial therapy and $23.74 \%$ among the patients without antimicrobial therapy. Blood culture positivity rate was significantly higher $(p<0.001)$ among the group without antimicrobial therapy. Our result was similar to Murty et al. (2007) in India who found $8.45 \%$ positive blood cultures in antibiotic user group and $38.89 \%$ in antibiotic non-user group.

In this study, $75 \%$ isolates of salmonella typhi were susceptible to ceftriaxone while resistance to ampicillin, cotrimoxazole and chloramphenicol were $43.75 \%, 56.25 \%$ and $50 \%$ respectively. Among them $43.75 \% \mathrm{~S}$. typhi isolates were multi drug resistant (MDR) but single drug resistant to ampicillin was (43.75\%), cotrimoxazole (56.25\%) and chloramphenicol (50.00\%). In a study by Chuang et al. 2008 reported $41.2 \%$ MDR S. typhi.

K. pneumoniae isolates in this study were $100 \%$ sensitive to imipenem and $75 \%$ to amikacin but $100 \%$ resistant to gentamicin, $75 \%$ resistant to cotrimoxazole, ciprofloxacin, ceftriaxone and cefixime each. These findings are almost similar to Dewanjee (2000) at BSMMU, Bangladesh.

Ceftriaxone and imipenem was the most effective (100\%) antimicrobial agent against E. coli. However, isolates were $100 \%$ resistant to ciprofloxacin, cefepime, amikacin, gentamicin, and netilmicin. Isolated S. marcescens were $100 \%$ sensitive to imipenem, cotrimoxazole and cefepime but $100 \%$ resistant to gentamicin and cefixime. Pseudomonas $\mathrm{sp}$. was $100 \%$ sensitive to amikacin and piperacillin-tazobactam but $100 \%$ resistant to ceftriaxone, cefixime, cotrimoxazole and imipenem. Ciprofloxacin and cefepime showed $66.67 \%$ sensitivity whereas $66.67 \%$ and $33.33 \%$ resistance to ceftazidime and gentamicin respectively. Almost similar sensitivity pattern was reported in Tanzania by Blomberg et al. (2007). On the other hand, A. baumannii, $75 \%$ sensitivity was noticed to ciprofloxacin \& imipenem each and $50 \%$ to both amikacin and Cotrimoxazole. Resistance was observed $100 \%$ against amoxyclavonic acid whereas ceftazidime, cefepime and gentamicin showed $75 \%$ each. 
Out of 233 suspected septicemia, 175 (75.11\%) patients were CRP positive (>6mg/l) and 58 (24.89\%) patients were CRP negative $(<6 \mathrm{mg} / \mathrm{l})$. Similarly in India, Makhija et al. (2005) found CRP positive in $84.3 \%$ cases of suspected septicemia patients. Culture proven septicemia (both blood culture and CRP positive) were 39 (16.74\%) cases, probable septicemia (only CRP positive) were 136 (58.37\%) and non-septicemic febrile patient (both blood culture and CRP were negative) were 58 (24.89\%) cases. Control children were sampled for baseline CRP levels measurement. Similar categorization was done in India by Bhartiya et al. (2000) and in France by Messer et al. (1996). In France, Messer et al. (1996) found 15.49\% cases of proven septicemia, $49.29 \%$ probable septicemia and $35.21 \%$ cases were non-septicemic febrile patient. This is consistent with our present study.

Mean serum CRP values of blood culture proven septicemia group, probable septicemia group, nonsepticemic febrile group and control group were $70.42 \mathrm{mg} / \mathrm{l}, 34.05 \mathrm{mg} / \mathrm{l}, 3.08 \mathrm{mg} / \mathrm{l}$ and $0.98 \mathrm{mg} / \mathrm{l}$ respectively. Statistically significant difference $(p<0.001)$ was found when mean CRP level of proven septicemia group, probable septicemia group and non-septicemic febrile group each compared with control group. None of the control group showed the concentration of CRP above cut-off level (cut-off value $6 \mathrm{mg} / \mathrm{l})$. On the other hand, it was found that culture proven septicemia group and probable septicemia group, both showed CRP level above cut-off value (cut-off value $6 \mathrm{mg} / \mathrm{l})$. CRP concentration were observed highly positive association ( $\mathrm{p}<$ 0.001) when compared among three different study groups.

We also attempted to find out the average age (mean \pm SD) of the culture proven septicemia, probable septicemia, non-septicemic febrile and control groups $(2098.23 \pm 1860.09,1093.75 \pm 1484.79,1011.44 \pm$ 1427.67 and $1631.8 \pm 1681.04$ days, respectively). No significant differences were found among the study groups with respect to age.

\section{Conclusion}

Most of the gram negative bacteria isolated from blood culture showed resistance to commonly used antibiotics. The high rate of antibiotic resistance of isolated organisms might be due to wide spread use of antibiotics. The most common causative pathogens are Salmonella sp., Klebsiella pneumoniae, Acinetobater baumannii and Pseudomonas sp. Since in this study CRP level is high (above $6 \mathrm{mg} / \mathrm{l}$ ) than normal range both in proven and probable septicemia group, so CRP may have a good biomarker tools in diagnostic and prognostic value. There is no significance found of CRP levels among age group of study cases. Blood culture and antimicrobial susceptibility must be done in every cases of suspected septicemia. Before starting of antimicrobial therapy, blood culture should be done.

\section{References}

Abucejo PE, Cepeding MR, Lupisan SP, Arcay J, Sombrero LT, Ruutu P, Herva E. 2001. Blood culture confirmed typhoid fever in a provincial hospital in the Philippines. Southeast Asian J trop Med Public Health 3, 531-536.

Ahmed NU, Chowdhury A, Houque M, Darmstadt GL. 2002.Clinical and bacteriological profile of neonatal septicemia in a tertiary level pediatric hospital in Bangladesh. Indian pediatr 39, 1034-1039.

Approved Standard NCCLS Doc M 7-A4.1966. Methods for antimicrobial susceptibility tests for bacteria that grow aerobically. (4 ${ }^{\text {th }}$ edn) Villanova, PA: National committee for clinical Laboratory Standards. 1998.

Bauer A, Kirby WMM, Sherries JC, Turck M. 1966. Antibiotic susceptibility testing by standardized single disc method. Am J Clin Pathol 45(5), 493-496.

Begum SA, Lutfor AB, Mollah AH, Hasan MK, Ahmed S, Akhter M, Salauddin NM. 2007. Salmonella- a new threat to neonates. Mymensingh Med J 16(2S), 15-18. 
Bhartiya D, Kapadia C, Sangvi K, Singh H, Kelkar R, Merchant R. 2000.Preliminary studies on IL-6 levels in healthy and septic Indian neonates. Indian pediatr 37, 1361-1367.

Blomberg B, Manji KP, Urassa KW, Tamim BS, Mwakagile DS, Jureen R, Msangi V, Tellevik MG, Petersen MH, Harthug S, Masselle SY, Langeland N. 2007. Antimicrobial resistance predicts death in Tanzanian children with bloodstream infections: A prospective cohort syudy. BMC infect Dis 7, 43. http://dx.doi.org/10.1186/1471-2334-7-43

Bont ED, Martens A, Rann JV, Samson G, Fetter W, Okken A, Leij LD, Kimpen J. 1994. Diagnostic value of plasma levels of tumour necrosis factor a (TNF $\alpha$ ) and interleukin- 6 (IL-6) in newborns with sepsis. Acta Paediatr 83, 696-699. http://dx.doi.org/10.1111/j.1651-2227.1994.tb13121.x

Brooks A W, Hossain A, Goswami G, Sharmeen AT, Nahar K, Alam K, Ahmed N, Naheed A, Nair GB, Luby S, Breimen RF. 2005. Bacteremic typhoid fever in children in an urban slum, Bangladesh. Emerg Infect Dis 11, 326-329. http://dx.doi.org/10.3201/eid1102.040422

Cheng AF, Fok TF, Duthie R, French GL. 1991. A five year prospective study of septicemia in hospitalized children in Hong Kong. J Trop Med Hyg 94, 295-303.

Chuang CH, Su LH, Perera J, Carlos C, Tan BH, Kumarasinghe G, So T, Van PH, Chongthaleong A, Hsueh PR, Liu JW,

Song JH, Chiu CH. 2008. Surveillance of antimicrobial resistance of Salmonella enterica serotype Typhi in seven Asian countries. Epidemiol Infect 1-4.

Dewanjee AK. 2000. A comparative study of different methods of blood culture for diagnosis of septicemia, BSMMU, Dhaka,

Esel D, Doganay M, Aip E, Sumerkan B. 2003. Prospective evaluation of blood cultures in a Turkish universities hospital: epidemiology, microbiology and patient outcome. J Clin Microbiol and Infect 9, 1038-1044. http://dx.doi.org/10.1046/j.1469-0691.2003.00714.x

Kreger BS, Craven DE, Carling PC, MC, McCabe WR. 1980. Gram negative bacteremia: Epidemiology and ecology in 612 patients. Ann J Med 68, 332-343. http://dx.doi.org/10.1016/0002-9343(80)90101-1

Laupland KB, Gregson DB, Zygun DA, Doig CJ, Mortis G, Church DL. 2004. Severe bloodstream infections: A population-based assessment. Crit Care Med 32 (4), 992-997. http://dx.doi.org/10.1097/01.CCM.0000119424.31648.1E

Makhija P, Yadav S, Thakur A. 2005.Tumour necrosis factor and interleukin-6 in infants with sepsis. Indian pediatric 42 , 1024-1028.

Messer J, Eyer D, Donato L, Gallatti H, Matis J, Simeoni U. 1996. Evaluation of interleukin-6 and soluble receptors of tumour necrosis factor for early diagnosis of neonatal infection. The J pediatr 129, 574-580. http://dx.doi.org/10.1016/S0022-3476(96)70123-3

Murphy PA. 1988. Septicemia. In: Weatherall DJ ed. Oxford text book of medicine. $2^{\text {nd }}$ ed. Oxford university press. Oxford. 608-613.

Murty DS, Gyaneshwari. 2007. Blood cultures in pediatric patients: A study of clinical impact. Indian J clin Microbial 25, 220-224.

Pierce G, Murray PR. 1986. Current controversies in the detection of septicemia. Eur J Clin Microbial 5, 487-491. http://dx.doi.org/10.1007/BF02017688

Rasul CH, Hassan MA, Habibullah M. 2007. Neonatal sepsis and use of antibiotic in a tertiary care hospital. Pak J Med Sci 23, 78-81. 
Saha SK, Baqui AH, Hanif M, Darmstadt GL, Ruhulamin, M, Nagatake T, Santhosum M, Black RE. 2001.Typhoid fever in Bangladesh: implications for vaccination policy. Pediatr Infect Dis J 20, 521-524. http://dx.doi.org/10.1097/00006454200105000-00010

Sharma NP, Peacock SJ, Phumratanaprapin W, Day N, White N, Pukrittayakamee S. 2006. A hospital-based study of bloodstream infections in febrile patients in Dhulikhel hospital Katmundu University teaching hospital, Nepal. Southeast Asian J Trop Med Public Health 37, 351-356.

Spencer RC. 1988. Blood cultures: where do we stand? J Clin Pathol 41, 668-670. http://dx.doi.org/10.1136/jcp.41.6.668

Washington JA II, Ilstrup DM. 1986. Blood cultures: Issues and controversies. Rev Infect Dis 8, 792-802. http://dx.doi.org/10.1093/clinids/8.5.792

Watson RS, Carcillo JA. 2005. Scope and epidemiology of pediatric sepsis. Pediatr Crit Care med 6, S3-S5. http://dx.doi.org/10.1097/01.PCC.0000161289.22464.C3

Weinstein MP, Murphy JR, Reller LB, Lichtenstein KA. 1983. The clinical significance of positive blood cultures: A comprehensive analysis of 500 episodes of bacteria and fungemia in adults. II. Clinical observations with special reference to factors influencing prognosis. Rev Infect Dis 5, 54-70. 